\title{
Social adversities and anxiety disorders in the Gaza Strip
}

\author{
A A M Thabet, P Vostanis
}

\begin{abstract}
Aim-To investigate the rate and nature of anxiety symptoms and disorders in children, and their relation to social adversities in a cultural sample not previously researched.
\end{abstract}

Methods-237 children aged 9 to 13 years living in the Gaza Strip were selected randomly from 112 schools. Children completed the revised manifest anxiety scale (a questionnaire with yes/no answers for 28 anxiety items and nine lie items), and teachers completed the Rutter scale (a questionnaire of 26 items of child mental health problems rated on a scale of 0-2: "certainly applies", "applies somewhat", “doesn't apply").

Results-Children reported high rates of significant anxiety problems $(21.5 \%)$ and teachers reported high rates of mental health problems in the children $(43.4 \%)$ that would justify clinical assessment. Anxiety problems, particularly negative cognitions, increased with age and were significantly higher among girls. Low socioeconomic status (father unemployed or unskilled worker) was the strongest predictor of general mental health problems. Living in inner city areas or camps, both common among refugees, was strongly associated with anxiety problems.

Conclusions-The rate and nature of anxiety disorders were similar to those established in Western societies. Factors reflecting social adversity and lack of stability were also similarly involved. There may be more similarities in the presentation of mental health symptoms across cultures than previously believed. (Arch Dis Child 1998;78:439-442)

Keywords: anxiety; mental health; deprivation; adversity

Gaza Community Mental Health Programme, PO Box 1049, Gaza, Palestine A A $M$ Thabet

Department of Child Psychiatry, University of Birmingham, Parkview Clinic, Queensbridge Road, Moseley, Birmingham B13 8QE, UK

$\mathrm{P}$ Vostanis

Correspondence to: Dr Vostanis.

Accepted 9 January 1998 children), low social class, family disruption and breakdown, father's employment (semiskilled or unskilled job), father's criminality, and school disadvantage have been associated with conduct disorders in children. ${ }^{2-4}$ Most research has found increased prevalence of conduct disorders in inner city, deprived areas. ${ }^{5}$ The identified socioeconomic risk factors are associated with poor child mental health outcome. $^{67}$
The association of anxiety (emotional) disorders with social adversity is less clear. ${ }^{7}$ Epidemiological studies in the UK and North America have found prevalence rates between $6 \%$ and $9 \%$ for severe conditions, including separation anxiety disorders, ${ }^{89}$ and about $20 \%$ for anxiety symptom constellations ${ }^{5}$ in the general population. In contrast with conduct disorders, there is some effect of socioeconomic status on the development of anxiety disorders, but this does not appear to be as strongly predictive as parental illness and life events. ${ }^{10}$

Research has predominantly been done in Western society ${ }^{11}$; it is unclear whether social adversity factors are similarly involved in the mechanisms underlying child psychopathology in cultures with different family and social structures. This study aimed to investigate the relation between social disadvantage and anxiety disorders among Palestinian children living in the Gaza Strip.

\section{Methods}

The Gaza Strip had a population of 860369 in 1995, excluding returnees from abroad after the peace process. The Gaza Strip has a high population density of 2150 people $/ \mathrm{km}^{2}$, which is a psychosocial risk factor for child psychopathology. The refugee population is $62.6 \%$. About $55 \%$ of the population live in eight crowded camps and $45 \%$ live in villages and towns. Half of the total population $(50.8 \%)$ are younger than 15 years. In 1995, the annual infant birth rate was $49.4 / 1000$ population, the infant mortality rate was between 26 and $50 / 1000$ infants, and the general population death rate was $8 / 1000$. Respiratory diseases and diarrhoea are major causes of infant morbidity and mortality. The annual increase of population growth is $4.5 \%$.

Twenty children aged of 9-13 years were randomly selected from each of 12 state schools in the five districts of the Gaza Strip. Ethics approval was granted by the Ministry of Education and parents gave informed consent before children were approached. For each child the Rutter teacher scale and the revised children's manifest anxiety scale (RCMAS) were administered.
RUTTER TEACHER SCALE

The Rutter teacher scale ${ }^{12} 13$ is a widely used and standardised screening questionnaire for the detection of child mental health problems in different cultures. It comprises 26 items of child mental health problems rated on a scale of 0-2: "certainly applies", "applies somewhat" or "doesn't apply". There are three subscales 
Table 1 Demographic characteristics of 237 children who completed the revised children's manifest scale

\begin{tabular}{lc}
\hline Age (years) & \\
Mean & 10.8 \\
Range & $9-13$ \\
Sex & $122(51.5 \%)$ \\
Male & $115(48.5 \%)$ \\
Female & \\
Family status & $228(96.2 \%)$ \\
Both parents & $7(3 \%)$ \\
Single mother & $2(0.8 \%)$ \\
Single father & \\
Father's employment & $63(27.4 \%)$ \\
Unemployed & $42(18.3 \%)$ \\
Unskilled worker & $48(20.9 \%)$ \\
Civil servant & $31(13.4 \%)$ \\
Professional & $46(20 \%)$ \\
Other & \\
Area of residence & $166(70 \%)$ \\
Urban & $44(18.6 \%)$ \\
Rural & $27(11.4 \%)$ \\
Camps &
\end{tabular}

measuring conduct, emotional, and hyperactivity problems. A score of 9 or above predicts a mental health disorder. The Rutter scales have been translated and piloted in Arabic.

REVISED CHILDREN'S MANIFEST ANXIETY SCALE The RCMAS ${ }^{13}$ is a standardised, 37 item, self report questionnaire for children aged 6-19 years. It measures the presence or absence of anxiety related symptoms (yes/no answers) in 28 anxiety items and nine lie items. Factor analysis of the anxiety items has identified three factors: physiological, worry/oversensitivity, and concentration. ${ }^{14}$ A cut off score of 18 predicts an anxiety disorder. ${ }^{15}$ This scale was also translated into Arabic and was completed in the presence of a researcher who clarified the items if necessary. We collected demographic data on family structure and family size, area of residence, housing status/overcrowding, and parents' employment status (table 1).

\section{Results}

Of the 240 children selected, 237 (98.7\%) agreed to take part (mean age 10.8 years). Almost all children (228) lived with both

Table 2 Presence of anxiety items (revised children's manifest anxiety scale) in 237 children aged 9 to 13 years

\begin{tabular}{lrl}
\hline Item/symptom & $n$ & $\%$ \\
\hline My hands feel sweaty & 107 & 45.1 \\
I have bad dreams & 99 & 41.8 \\
I get nervous when things do not go the right way for me & 90 & 38.0 \\
Others seem to do things easier than I can & 84 & 35.4 \\
It is hard to get to sleep at night & 80 & 33.8 \\
My feelings get hurt easily when I am fussed at & 79 & 33.3 \\
I get "mad" easily & 76 & 32.1 \\
I worry about what is going to happen & 75 & 31.6 \\
I have trouble making up my mind & 75 & 31.6 \\
I am afraid of a lot of things & 71 & 30.0 \\
My feelings get hurt easily & 71 & 30.0 \\
Other children are happier than I & 66 & 27.8 \\
Often I have trouble getting my breath & 62 & 26.2 \\
I worry about what other people think about me & 58 & 24.5 \\
I wake up scared some of the time & 57 & 24.1 \\
I worry about what my parents will say to me & 54 & 22.8 \\
I worry when I go to bed at night & 52 & 21.9 \\
I am nervous & 51 & 21.5 \\
I often worry about something bad happening to me & 51 & 21.5 \\
I wiggle in my seat a lot & 50 & 21.1 \\
I feel alone even when there are people with me & 47 & 19.8 \\
It is hard to keep my mind on my school work & 47 & 19.8 \\
I worry a lot of the time & 46 & 19.4 \\
I feel someone will tell me I do things the wrong way & 46 & 19.4 \\
I feel that others do not like the way I do things & 40 & 16.9 \\
Often I feel sick in my stomach & 40 & 16.9 \\
A lot of people are against me & 33 & 13.9 \\
I am tired a lot & 17 & 7.2 \\
\hline
\end{tabular}

parents (96.2\%) and 217 children (91.6\%) had at least three siblings. Only one child had lived in care, and only 12 mothers were employed outside the home.

The most frequent abnormal behaviours indicated by teachers (rated as "certainly applying") were, bullying (11\%), lying $(10.5 \%)$, disobedience $(10.5 \%)$, and fighting $(6 \%)$. The most frequent emotional items were worries $(17.3 \%)$, fears $(11.8 \%)$, and feeling miserable $(5.9 \%)$. The most frequent hyperactivity items were restlessness $(16.0 \%)$, poor concentration $(12.2 \%)$, and being squirmy (11.4\%). Significant school absence related to anxiety was reported in 21 children $(8.9 \%)$, and truancy related to behaviour problems in six children (2.5\%). Many children (104, $43.4 \%$ ) exceeded the cut off point of 9, which indicates the presence of significant mental health problems. Boys had higher scores on the hyperactivity subscale than girls (MannWhitney $U$ test: $z=2.07, p=0.04)$. Low socioeconomic status (father being unemployed or unskilled worker) was the sociodemographic variable that best predicted the presence of mental health problems in children in a forward stepwise logistic regression $(\mathrm{B}=0.38, \mathrm{SE}=0.13, \mathrm{p}=0.004)$. This variable also best predicted the total conduct subscale score (linear regression; $\mathrm{B}=1.01,95 \%$ confidence intervals (CI) 0.34 to 1.68 , $\mathrm{p}=0.003)$. The total teacher rated emotional subscale score was best predicted by the age of the child (increased with age; $\mathrm{B}=0.67,95 \%$ CI 0.02 to $1.32, \mathrm{p}=0.04$ ).

Table 2 shows the self reported anxiety items of the RCMAS). Fifty one children (21.5\%) scored above the cut off score for anxiety disorders. Girls reported significantly higher total anxiety scores (mean 8.2) than boys (mean 6.5) (Mann-Whitney $\mathrm{U}$ test $\mathrm{z}=2.44$, $\mathrm{p}=0.015)$, and they were significantly more likely to have scores above the cut off $\left(\chi^{2}=3.9\right.$, $\mathrm{df}=1, \mathrm{p}=0.047)$. Girls also scored significantly higher than boys on most anxiety items particularly: "having trouble making up my mind" (Mann-Whitney $U$ test $\mathrm{z}=2.9$, $\mathrm{p}=0.003)$; "getting nervous when things do not go the right way" $(\mathrm{z}=2.2, \mathrm{p}=0.03)$; "others do things easier than me" $(\mathrm{z}=2.2$, $\mathrm{p}=0.03)$; "worrying about what parents will say" ( $z=2.1, p=0.03)$; "feeling that others do not like the way I do things" $(z=2.3$, $\mathrm{p}=0.02)$; sickness $(\mathrm{z}=3.3, \mathrm{p}=0.001)$; "impaired concentration" $(\mathrm{z}=2.0, \mathrm{p}=0.04)$; and "often worrying about something bad happening" $(\mathrm{z}=3.3, \mathrm{p}=0.001)$.

Children with possible anxiety disorders were more likely to be living in an inner city area or camps $\left(\chi^{2}=9.2, \mathrm{df}=2, \mathrm{p}=0.01\right)$. Older age $(\mathrm{B}=0.33, \mathrm{SE}=0.12, \mathrm{p}=0.01)$ and living in inner city or camps $(B=0.59$, $\mathrm{SE}=0.3, \mathrm{p}=0.05$ ) were the strongest predictors of anxiety disorders in a logistic regression. Teacher rated conduct and hyperactivity scores were strongly correlated $(r=0.76$, $\mathrm{p}<0.0005)$.

We compared the responses of the youngest (9 years; $n=62$ ) with oldest ( 13 years; $n=26$ ) children (Mann-Whitney U test) to investigate 
the potential change of anxiety symptoms with age; older children scored significantly higher on seven anxiety symptoms, mainly related to cognitions.

\section{Discussion}

We found a similar pattern of anxiety symptoms and disorders among children living in the Gaza Strip to previous epidemiological research from Western societies. There were high rates of anxiety disorders and school related mental health problems. In a previous community survey in the United States using the RCMAS, Kashani and Orvaschel ${ }^{16}$ found the same prevalence rate $(21 \%)$ of anxiety disorders, which included separation anxiety, phobic, and overanxious disorders. Our study identified similar frequencies of symptoms, such as worries and nightmares, to the American study.

Anxiety problems significantly increased with age, particularly among girls. Although "physiological" symptoms of anxiety were frequently reported (insomnia, nightmares, sweating), children also experienced a substantial amount of negative cognitions related to poor self image and self esteem. Social cognitions, such as self awareness, shame and guilt, first develop in middle childhood (7-8 years). Between the ages of 9 and 12 years, children can distinguish between conflicting emotions, and between others' accidental and intentional behaviours. Internal psychological factors are increasingly perceived as causal to the child's behaviour. During adolescence, social cognitions are enhanced further, together with the development of belief systems, hypothetical and abstract thinking, and self evaluation of their thought process. This explains the increase of anxiety related cognitions with age, which is consistent with previous findings, ${ }^{16}$ and has implications for interventions, particularly cognitive behavioural treatment. ${ }^{17}$

Our findings did not support the commonly held belief that, in non-Western societies, anxiety and other mental health symptoms are predominantly expressed through somatising symptoms. This view may be because of a lack of systematic epidemiological research on cross cultural issues. Studies on phenomenology indicate that child mental health symptoms do not differ significantly across cultures and that culture specific mental health disorders are rare. ${ }^{18}$

Social adversity factors were implicated in the development of anxiety and other mental health problems, even within a society with extended family and community networks. As in earlier UK based research, ${ }^{34}$ these factors were lower socioeconomic status (father's employment status) and living in inner city areas. A mediating factor could be the social instability of refugee families within this population who lived in urban areas and camps. In contrast, the social support system of families from rural areas has not been disrupted despite the war, and this may have acted as a protective factor for the children. In a study of Croatian children during the war, Zivcic ${ }^{19}$ found signifi- cantly higher depressive and phobic symptoms in displaced (refugee) than in local children in stable social conditions. In contrast with studies from Western countries, large family size (overcrowding) was not associated with child mental health problems. In Arabic families, a large number of children may not be an index of adversity or deprivation.

Children's experience of traumas in war zones is often directly related to the development of anxiety symptoms such as fear and nightmares. In a prevalence study of posttraumatic stress disorders (PTSD) reactions from another young population living in the same area, we found PTSD reactions of at least mild severity in $73 \%$ of children aged 6-11 years, and $39 \%$ of the children reported moderate to severe PTSD reactions (unpublished data). Other studies of children of war have found similar associations between trauma and psychopathology. Ahmad ${ }^{20}$ established PTSD in about $25 \%$ of displaced Kurdish children, and Weine and colleagues ${ }^{21}$ found similar rates in Bosnian adolescents who had moved to America during the war. Nader and colleagues $^{22}$ established moderate to severe post-traumatic stress reactions in $70 \%$ of Kuwaiti children following the Gulf war.

Limitations of our study need to be acknowledged, particularly the lack of diagnostic interview and corroborative information from parents. Research instruments used in cross cultural research need further development, as little is known about potential cultural influences on how children interpret certain questions. ${ }^{23}$ Future research also needs to address the potential impact of family and social beliefs and perceptions of child mental health problems. Child mental health services in developing countries are often best integrated with existing primary child health care services. ${ }^{18}$ Treatment intervention programmes need to be developed and evaluated for traumatised children.

We are grateful to all the children and teachers who participated in the study as well as to Dr Saeed Haque for statistical advice.

1 Pearce J. Child health surveillance for psychiatric disorder. Arch Dis Child 1993;69:394-8.

2 Rutter M. Why are London children so disturbed? Proc Roy Soc Med 1973;66:53-7.

3 Rutter M, Yule B, Quinton D, Rowland O, Yule W, Berger M. Attainment and adjustment in two geographical areas: III. Some factors accounting for area differences. $B r \not P P_{s y}$ chiatry 1975;126:520-33.

4 Kolvin I, Miller F, Scott D, Gatzanis S, Fleeting M. Continuities of deprivation? The Newcastle 1000 family study.

5 Rutter M. Isle of Wight revisited: twenty-five years of child psychiatric epidemiology. 7 Am Acad Child Adolesc Psychiapsychiatric epidemio

6 Kolvin I, Miller F, Fleeting M, Kolvin P. Social and parenting factors affecting criminal-offence rates. Brf Psychiatry 1988;152:80-90

7 Velez C, Johnson J, Cohen P. A longitudinal analysis of selected risk factors for childhood psychopathology. $\mathcal{f} \mathrm{Am}$ Acad Child Adolesc Psychiatry 1989;28:861-4.

8 Bowen R, Offord D, Boyle $M$. The prevalence of overanxious disorder and separation anxiety disorder: results from the Ontario Child Health Study. $\mathcal{F}$ Am Acad Child Adolesc Psychiatry 1990;29:753-8.

9 Bernstein G, Borchardt C, Perwien A. Anxiety disorders in children and adolescents. F Am Acad Child Adolesc Psychiatry 1996;35:1110-19.

10 Beidel D, Turner S. At risk for anxiety: I. Psychopathology in the offspring of anxious parents. $\mathcal{F}$ Am Acad Child Adolesc in the offspring of anxious

11 Bird H. Epidemiology of childhood disorders in a cross-cultural context. F Child Psychol Psychiatry 1996;37: 35-49. 
12 Rutter M, Tizard J, Whitmore K. Education, health and behaviour. London: Longman, 1970.

13 Reynolds C. Concurrent validity of what I think and feel: the revised children's manifest anxiety scale. $\mathcal{F}$ Consult Clin Psychol 1980;48:774-5.

14 Reynolds C, Paget K. Factor analysis of the revised children's manifest anxiety scale for blacks, whites, males and females with a national normative sample. $f$ Consult Clin Psychol 1981;49:352-9.

15 Montgomery LE, Finch AJ. Validity of two measures of anxiety in children. $\mathcal{F}$ Abn Child Psychol 1974;12:293-7.

16 Kashani JH, Orvaschel $\mathrm{H}$. A community study of anxiety in children and adolescents. Am f Psychiatry 1990;147:313318.

17 Vostanis P, Harrington R. Cognitive-behavioural treatment of depressive disorder in child psychiatric patients European Child and Adolescent Psychiatry 1994;3:111-23.

18 Nikapota A. Child psychiatry in developing countries. $\mathrm{Br} f$ Psychiatry 1991;158:743-51.
19 Zivcic I. Emotional reactions of children to war stress in Croatia. I Am Acad Child Adolesc Psychiatry 1993;32:70913.

20 Ahmed A. Symptoms of posttraumatic stress disorder among displaced Kurdish children in Iraq: victims of a man-made disaster after the Gulf war. Nordic fournal of Psychiatry 1992;46:314-19.

21 Weine S, Becker D, McGlashan T, Vojvoda D, Hartman S, Robbins J. Adolescent survivors of "ethnic cleansing" on the first year in America. 7 Am Acad Child Adolesc Psychiathe first year in Ame

22 Nader K, Pynoos R, Fairbanks L, Al-Ajeel M, Al-Asfour A. A preliminary study of PTSD and grief among the children of Kuwait following the Gulf crisis. Br F Clin Psychol 1993; 32:407-16.

23 Dong Q, Yang B, Ollendick T. Fears in Chinese children and adolescents and their relations to anxiety and depression. $f$ Child Psychol Psychiatry 1994;35:351-63. 